\title{
Shear thickening and scaling of the elastic modulus in a fractal colloidal system with attractive interactions
}

\section{Citation}

Osuji, Chinedum 0., Chanjoong Kim, and David A. Weitz. 2008. "Shear Thickening and Scaling of the Elastic Modulus in a Fractal Colloidal System with Attractive Interactions." Physical Review E77 (6): 060402. https://doi.org/10.1103/PhysRevE.77.060402.

\section{Permanent link}

http://nrs.harvard.edu/urn-3:HUL.InstRepos:41511280

\section{Terms of Use}

This article was downloaded from Harvard University's DASH repository, and is made available under the terms and conditions applicable to Other Posted Material, as set forth at http:// nrs.harvard.edu/urn-3:HUL.InstRepos:dash.current.terms-of-use\#LAA

\section{Share Your Story}

The Harvard community has made this article openly available. Please share how this access benefits you. Submit a story.

Accessibility 


\title{
Shear thickening and scaling of the elastic modulus in a fractal colloidal system with attractive interactions
}

\author{
Chinedum O. Osuji, ${ }^{*}$ Chanjoong Kim, and David A. Weitz \\ School of Engineering and Applied Sciences, Harvard University, Cambridge, Massachusetts 02138, USA
}

(Received 23 October 2007; revised manuscript received 27 March 2008; published 11 June 2008)

\begin{abstract}
Dilute oil dispersions of fractal carbon black particles with attractive van der Waals interactions display continuous shear thickening followed by shear thinning at high shear rates. The shear thickening transition occurs at $\dot{\gamma}_{c} \approx 10^{2}-10^{3} \mathrm{~s}^{-1}$ and is driven by hydrodynamic breakup of clusters. Pre-shearing dispersions at shear rates $\dot{\gamma}>\dot{\gamma}_{c}$ produces enhanced-modulus gels where $G^{\prime} \sim \sigma_{\text {pre-shear }}^{1.5-2}$ and is directly proportional to the residual stress in the gel measured at a fixed sample age. The observed data can be accounted for using a simple scaling model for the breakup of fractal clusters under shear stress.
\end{abstract}

DOI: 10.1103/PhysRevE.77.060402

PACS number(s): 82.70.Dd, 82.70.Gg, 83.60.Rs

Colloidal particles interacting with attractive potentials in a fluid form gels above some critical volume fraction, $\phi_{c}$, where $\phi_{c}$ is a function of the interaction strength, $U$. The viscoelastic properties of the gels are determined by $\phi, U$, and the topology of the network of particle contacts, characterized by a fractal dimension, $d_{f}$. Additionally, the properties are sensitive to the mechanical history of the system. To obtain reproducible results, rheological studies of colloidal gels typically employ a high rate pre-shear as a rejuvenation or initialization step in which the mechanical history of the gel is effectively erased. The non-Newtonian flow curves associated with attractive colloidal suspensions typically display monotonic shear thinning and as a result the modulus established after rejuvenation is not overly sensitive to the exact pre-shear flow condition. Shear thickening transitions are neither generally known nor predicted for systems where attractive interactions are sufficiently strong to induce flocculated gels $[1,2]$. Such transitions would be accompanied by drastic changes in the underlying fluid microstructure. As a result, the elasticity of gels would show a marked dependence on the nature of the pre-shear flow and its location on the flow curve relative to the shear thickening regime.

Here we report on the observation of shear thickening for attractive colloidal particles in a simple Newtonian fluid. We find the elasticity of gels formed by pre-shearing above the shear thickening transition scales as a power law with the pre-shear stress, and is directly proportional to the internal stress in the sample, which we measure. We propose a scaling model which considers the dependence of the gel modulus on the cluster number density produced during pre-shear flow. The model shows good agreement with the experimental data.

Measurements were made at $25^{\circ} \mathrm{C}$ on dispersions of carbon black particles ranging from 2 to $8 \mathrm{wt} . \%$. A nonpolar, small-molecule fluid, tetradecane, was used as the suspending medium to avoid complicating influences of electrostatics or adsorption of macromolecular solvent species onto the colloidal surface. The carbon black (Cabot Vulcan XC72R) exists as $\approx 0.5 \mu \mathrm{m}$ diameter fumed particles with fractal di-

\footnotetext{
*chinedum.osuji@yale.edu. Present Address: Department of Chemical Engineering, Yale University, New Haven, CT 06511.
}

mension $d_{f}^{p}=2.2$ and $\rho_{\text {carbon }}=1.8 \mathrm{~g} / \mathrm{cm}^{3}$. Tetradecane $\left(\rho_{\text {solvent }}=0.762 \mathrm{~g} / \mathrm{cm}^{3}, \quad \eta_{\text {solvent }}=2.8 \mathrm{mPa} \mathrm{s}\right)$ was obtained from Aldrich Chem. Co. Samples were prepared by vigorous mixing of the two components using a vortexer. Optical studies were conducted using a Bohlin CS rheometer with a transparent base plate through which samples were imaged using a charge-coupled device (CCD) camera. Rheological measurements were made in strain-control mode using a TA Instruments AR-G2 rheometer with cone-plate and doublewall Couette geometries. The fast hardware feedback of this instrument, when run in strain control, enabled measurements of the internal stress within samples by applying continuously computed torques to maintain constant tool position. Samples were pre-sheared before each measurement to remove the effect of shear history using an empirically determined shear rate $\left(100 \mathrm{~s}^{-1}\right)$ which provided consistent results [3]. Samples were checked using various geometries (standard and roughened) to ensure the absence of rheological artifacts and phenomena such as wall slip and edge fracture. They were also replicated using a pure strain-controlled instrument, ARES-LS1 (Rheometrics).

We measure the steady state viscosity as a function of shear rate using a cone-plate geometry. At low shear rates, between $\approx 10^{0}$ and $10^{1} \mathrm{~s}^{-1}$, optical studies using the plateplate geometry show a buildup of structure in the sample via the formation of rolling, vorticity aligned cylindrical flocs. Such shear-induced structures have been observed in other viscoelastic systems under flow such as thixotropic clay gels [4], nanotube suspensions [5], and attractive emulsion droplets [6]. Viscometry of such "sticky" systems at low shear rates may be susceptible to wall slip effects [7] and so caution is required in interpreting any rheological data in this regime. At higher shear rates where homogeneous flows are readily achieved, $\dot{\gamma}>10 \mathrm{~s}^{-1}$, Fig. 1, there is modest shear thinning accompanied by the formation of densified clusters, as shown in Fig. 2(a). Shear thinning persists until $\approx 10^{2}-10^{3} \mathrm{~s}^{-1}$ where there is a composition dependent continuous transition to a shear thickening flow. The flow curves exhibit a hysteresis loop on descending sweeps as is typical of thixotropic materials, and show good reproducibility on subsequent ascending-descending loops. Shear thickening has in fact been reported [8], though not extensively considered, for a system consisting of carbon black particles in an 


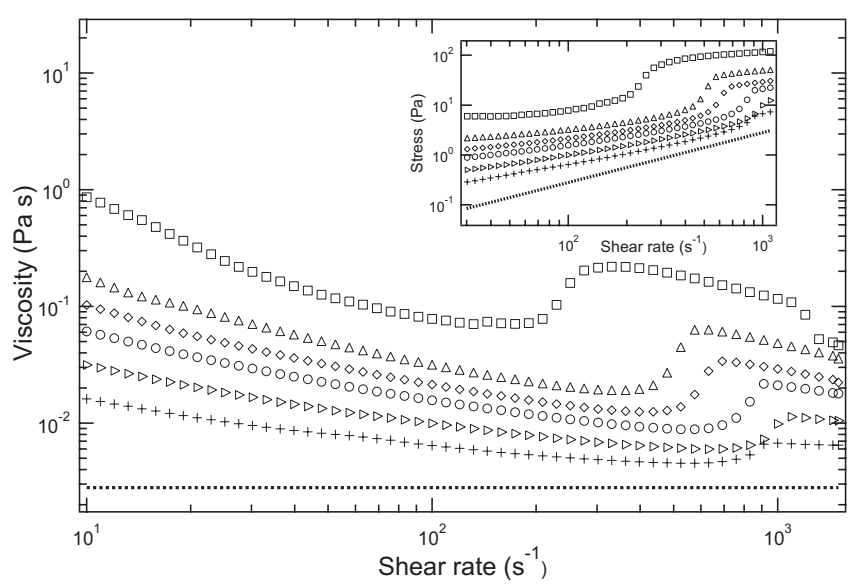

FIG. 1. Shear rate dependent viscosity for a series of weight fractions. +: $2 \%, \triangleright: 3 \%, \bigcirc: 4 \%, \diamond: 5 \%, \triangle: 6 \%, \square: 8 \%$. The dotted line is the background viscosity of the solvent. Inset: Rate dependent shear stress.

adsorbing silicone oil. This was attributed to hydrocluster formation due to the occurrence of stress overshooting at shear rates that produced shear thickening. In the present case, at high shear rates in the shear thickening regime, the system presents a finely dispersed microstructure in which the densified clusters produced at lower shear rates have been eroded. This results in a higher effective volume fraction of particles in the dispersion, and thus an enhanced viscosity, Fig. 2(b). In contrast to the hard sphere case, shear thickening results not from hydrocluster formation, but from the breakup of locally dense clusters of the fractal colloidal particles into less dense structures which increase viscous dissipation in the system. A negative thixotropy of ferricoxide suspensions composed of acicular particles has also been attributed to a qualitatively similar mechanism [9].

Based on the dispersed particle size, we estimate the Péclet number, $\mathrm{Pe}=\frac{\sigma a^{3}}{k_{B} T} \approx 10^{2}-10^{3}$, confirming that the high shear rate regime is dominated by hydrodynamic forces. Similarly, the particle Reynold's number $\operatorname{Re}=\frac{\rho \dot{\gamma} a^{2}}{\eta}$ $\approx 10^{-4}-10^{-5}$, indicating that viscous effects dominate and contributions from inertial forces should be minimal. Under similar conditions in Stokesian dynamics simulations of hard spheres $[10,11]$, a transition is observed from positive to negative normal stresses on shear thickening due to hydrocluster formation at high Pe, with $\left|N_{1}\right| \sim \dot{\gamma}^{1}$ where $N_{1}$ is the

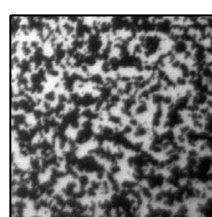

a

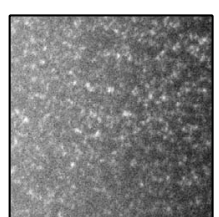

b
FIG. 2. Microstructure under shear in parallel plate geometry. Gap $=100 \mu \mathrm{m}, \phi=3$ wt. $\%, 1.5 \times 1.5 \mathrm{~mm}$ area. (a) $\dot{\gamma}=133 \mathrm{~s}^{-1}$ (b) $\dot{\gamma}=1330 \mathrm{~s}^{-1}$. Illumination for (b) was several times higher than for (a) to obtain sufficient light transmission through the optically dense structure. Pixel binning was used to decrease the required exposure time.

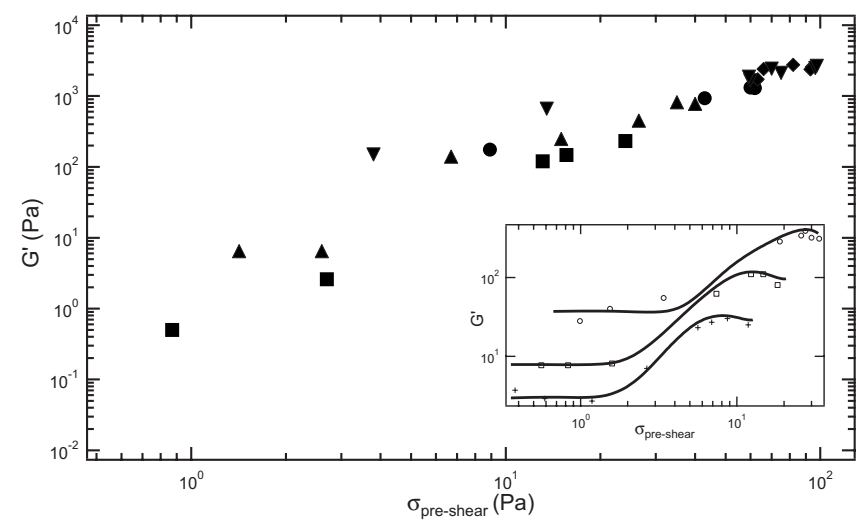

FIG. 3. Elasticity of shear thickened gels as a function of the pre-shear stress, using cone-plate geometry. $\mathbf{\square}: 3 \%, \mathbf{\Delta}: 4 \%, \mathbf{0}: 5 \%$, $\checkmark$ : $6 \%, \boldsymbol{\nabla}: 8 \%$. Inset: Data obtained spanning low and high stresses using a double-wall Couette cell. $+: 2 \%, \square: 3 \%, \bigcirc: 5 \%$.

first normal stress. In the present system, inertia corrected $N_{1}$ transitioned to slight net positive values at the onset of shear thickening [12], suggesting, along with the optical data, that hydrocluster formation is not in fact the mechanism in operation here. The critical shear rate at the onset of shear thickening, $\dot{\gamma}_{c}$, scales roughly as $\phi^{\beta}$ and the critical stress $\sigma_{c}$ as $\phi^{\nu}$ with $\beta$ roughly between -1.5 and -2 , and $\nu \approx 1$. From measurements performed using high viscosity basestock oil $(\eta \approx 33 \mathrm{mPa} \mathrm{s}$ ), we observe that the critical shear rate is inversely proportional to the solvent viscosity, suggesting that shear thickening in this system is controlled by a critical stress, rather than by a critical rate.

We use dynamic measurements to study the dependence of gel modulus on pre-shear flow and composition. Samples were sheared to remove the effects of flow history and then pre-sheared at the rate of interest for $20 \mathrm{~min}$, more than sufficient to achieve a steady flow and constant viscosity. The system was then allowed to sit quiescently for 30 minutes. We measure the viscoelastic storage and loss moduli, $G^{\prime}$ and $G^{\prime \prime}$, in the linear regime, $\gamma=0.05 \%$ at a fixed angular frequency $\omega=1 \mathrm{rad} / \mathrm{s}$, where $\gamma$ is the shear strain. The elastic modulus of gels formed by pre-shearing at a fixed rate in the shear thickening regime follows a typical power law $G^{\prime}$ $\sim \phi^{\alpha}$, with exponent $\alpha=3.5$, as might be expected for attractive systems [13]. In the shear-thinning regime, before the thickening transition, the exponent is slightly higher, $\alpha=4.2$. The elasticity of thickened gels is strongly enhanced, typically by over one order of magnitude, compared to gels produced by non-shear-thickening flow. This suggests that in addition to volume fraction, $\phi$, there is another parameter dependence involved in determining the elasticity of gels produced across a range of shear rates. The modulus follows the same qualitative dependence on shear rate as the viscosity, with a sudden upturn at the critical rate or critical shear stress. A double-wall Couette cell was used to mitigate the effects of sedimentation at low shear stresses where samples did not form robust thickened gels, or were too dilute to be gravitationally stable for study in the cone-plate geometry. Remarkably, as in Fig. 3 (inset), the dependence of the gel modulus on the pre-shear stress has a common form across different compositions, $\phi$, such that the data can be scaled 


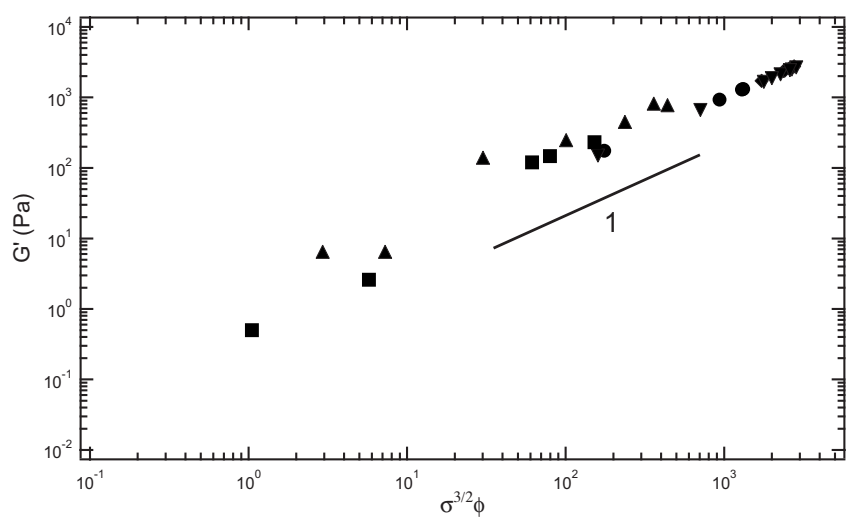

FIG. 4. Elasticity of shear thickened gels as a function of the pre-shear stress rescaled according to Eq. (2) with $d_{f}=1.8$. $\mathbf{\square}: 3 \%$, $\boldsymbol{\Delta}: 4 \%, \mathbf{0}: 5 \%, 6 \%, \boldsymbol{\nabla}: 8 \%$. The solid line is a visual guide with a slope of 1 .

onto a single curve using the critical stress, $\sigma_{c}$, and critical modulus, $G_{c}^{\prime}$, as scaling parameters. Data of shear thickened samples show a striking dependence of the modulus on the pre-shear stress, $G^{\prime} \sim \sigma^{1.5-2}$, as shown in Fig. 3 (main). These samples were studied in the cone-plate geometry at large pre-shear stresses where the resulting high modulus gels were not sensitive to sedimentation. There is a somewhat weaker volume fraction dependence, such that in the shear thickening regime, overall, the modulus of the colloidal gel is substantially influenced by the pre-shear stress applied to the system prior to gelation.

We interpret these results in terms of the dependence of the cluster size on shear stress. As the stress increases, densified clusters break and assume a size that is set by the balance between the shear stress and the cohesive energy density of the cluster.

The shear force on the cluster, $F_{\text {shear }}=4 \pi \sigma R_{c}^{2}$ where $R_{c}$ is the radius of the fractal cluster. This force is spread across $N_{s}$ particles in the plane of shear of the cluster is given by the following expression, $N_{s} \sim R_{c}^{d_{f}-1}$, where $d_{f}$ is the fractal dimension of the cluster. This force is balanced by the attractive interaction between particles, defined by the interaction strength $U$ and the relevant length scale, $a$, the particle diameter. Thus we have

$$
R_{c}^{d_{f}-3} \sim \frac{4 \pi \sigma a}{U},
$$

which sets the dependence of the equilibrium cluster size on the shear stress. The elastic modulus scales directly with the number density of clusters, i.e. $G^{\prime} \sim \nu U$, the cohesive energy density, as in many disordered systems [14]. The number density $\nu$ of clusters of radius $R_{c}$ scales as $\phi / R_{c}^{d_{f}}$, so that overall we expect the gel modulus to scale as

$$
G^{\prime} \sim \phi \sigma^{d_{f} / 3-d_{f}} .
$$

We assume a cluster fractal dimension of 1.8 , as is found in diffusion limited aggregating systems then $G^{\prime} \sim \sigma^{3 / 2} \phi$. We plot this in Fig. 4 and find good accord between our data and a slope of 1 as shown over several decades. Our scaling is in rough agreement with empirical data on flocculated systems

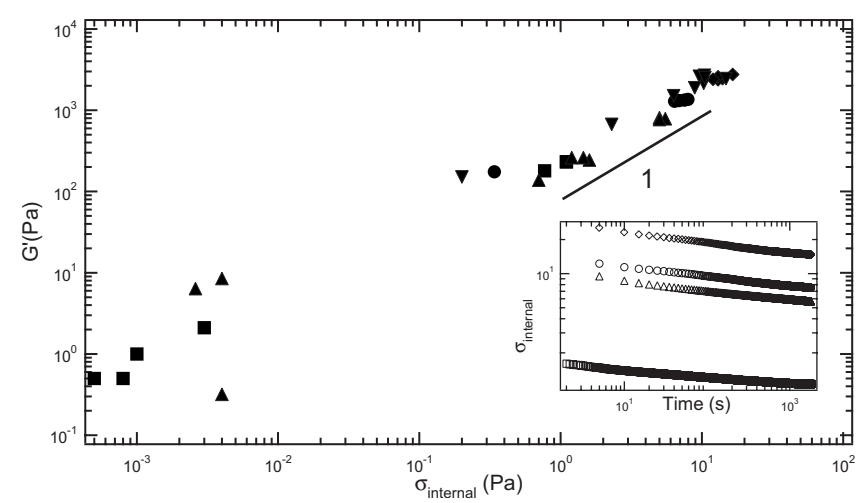

FIG. 5. Shear modulus scales linearly with residual stresses resulting from the quench into the gel state on cessation of flow. $\mathbf{\square}$ $3 \%, \mathbf{\Delta}: 4 \%, \mathbf{0}: 5 \%, 6 \%, \boldsymbol{\nabla}: 8 \%$. The solid line is a visual guide with a slope of 1 . Inset: Time dependence of internal stress, with open symbols for clarity.

where the number of particles in a floc scales roughly as $N_{c} \sim \sigma^{-1}$ [15]. Although the simple assumptions of the scaling argument provide satisfactory results, the choice for $d_{f}$ should be rationalized by more detailed measurements than we have so far made.

The abrupt cessation of shear in a freely flowing system above $\phi_{c}$ results in a sudden entry into the gel state. The system loses mobility as particles experience sticky contacts with neighbors, leading to the formation of structures that rapidly span space, forming an elastic medium. This rapid sol-gel transition should be characterized by the development of internal or residual stresses, $\sigma_{i}$, coincident with the mechanical quench - the network structure, deformed with respect to an equilibrium relaxed configuration, would exhibit stresses which relax gradually in time. These stresses are believed to drive the peculiar dynamics observed in the aging of some glassy systems $[16,17]$. Since they result from a deformation of the structure, the internal stresses are expected to be proportional to the elastic modulus [18], though no direct experimental evidence as yet confirms this. Previous work has examined the very short time $(t \lesssim 0.1 \mathrm{~s}) \mathrm{dy}-$ namics of stresses in weakly flocculating systems subjected to stress jumps [19]. Here, we followed $\sigma_{i}(t)$ in our system from $t \approx 1 \mathrm{~s}$ after cessation of flow to $t \approx 30 \mathrm{~min}$ and find a weak power law decay over this period. For shear thickened gels, the stress scales roughly as $\sigma_{i} \sim t^{-0.1}$ (inset Fig. 5). The internal stress acts counter to the direction in which the sample was pre-sheared and is reversed on changing the preshear direction, indicating that the deformation of the clusters during flow (relative to the quiescent state) gives rise to the residual stress that is manifested by the rate quench and rapid gelation. Critically, we find that the internal stress at a fixed sample age, $t=30 \mathrm{~min}$, is directly proportional to the modulus of the system measured immediately thereafter, over several orders of magnitude of internal stress, produced by different pre-shear flows (Fig. 5). The prototypical picture of a slow diffusion limited gelation clearly does not apply despite the relatively low volume fractions under study here. Gelation is unusually rapid and the system displays a welldeveloped elastic modulus at the shortest times studied, $\approx 1 \mathrm{~s}$ after cessation of shear. 
Shear thickening in hard sphere and repulsive Brownian systems has been rationalized using a force balance model that compares the shear force pushing particles together to the diffusive flux that inhibits cluster formation [20].

$$
\frac{3 \pi \mu \dot{\gamma}_{c} a^{3}}{2 h}=-k_{B} T \frac{\partial \ln g(r)}{\partial r},
$$

where $a$ is the particle radius, $h=r-2 a$ the distance between two particles, $g(r)$ the particle distribution function, and $\mu$ the viscosity of the medium, replaceable by the suspension viscosity, $\eta$, as a way of accommodating many body effects. In the system under study here, at low shear stresses, large non-Brownian clusters resulting from breakage of the gel at low shear rates have no diffusive barrier towards further clustering. They densify as shear stresses force them into contact, reducing their effective volume fraction in the system. Above a critical stress, however, the cohesive energy density of the clusters is overcome and they start to disintegrate with a attendant rise in viscosity.

The observation of shear thickening in this system of attractive particles was surprising. Indeed, previous careful examinations of flocculated systems have not resulted in observation of shear thickening in fractal [2] and "solid" particulate systems [1]. A particularly systematic examina- tion of the role of interaction strength using depletion forces [1] shows that shear thickening diminished and eventually disappeared on addition of substantial attractive interactions. It is clear that in the current system, the fractal nature of the particles, irreversibly fused agglomerates of smaller particles produced by flame pyrolysis, plays a key role. It drives the increase in the effective volume fraction on cluster break-up. Shear thickening is thus a product of shear induced reduction of cluster size and increase of the effective volume fraction of the particles in the dispersion due to the fractal particle structure. Correspondingly, the modulus of shear thickened dispersions is enhanced due to the increase in cluster number density. The scaling of the elastic modulus with pre-shear stress is satisfactorily accounted for by consideration of the equilibrium cluster size as a function of this stress. The direct proportionality between the elastic modulus and the internal stress resulting from a system quench on cessation of shear has been confirmed by direct measurement. The dynamics of these internal stresses are a topic for continued studies.

The authors gratefully acknowledge L. Cipelletti, V. Trappe, and H. Wyss for helpful discussions, A. Negi for supplementary rheological measurements and the Infineum Corporation for funding.
[1] V. Gopalakrishnan and C. F. Zukoski, J. Rheol. 48, 1321 (2004).

[2] S. R. Raghavan and S. A. Khan, J. Colloid Interface Sci. 185, 57 (1997).

[3] V. Trappe and D. A. Weitz, Phys. Rev. Lett. 85, 449 (2000).

[4] F. Pignon, A. Magnin, and J. M. Piau, Phys. Rev. Lett. 79, 4689 (1997).

[5] S. Lin-Gibson, J. A. Pathak, E. A. Grulke, H. Wang, and E. K. Hobbie, Phys. Rev. Lett. 92, 048302 (2004).

[6] A. Montesi, A. A. Pena, and M. Pasquali, Phys. Rev. Lett. 92, 058303 (2004).

[7] H. Walls, S. Caines, A. Sanchez, and S. Khan, J. Rheol. 47, 847 (2003).

[8] M. Kawaguchi, M. Okuno, and T. Kato, Langmuir 17, 6041 (2001).

[9] H. Kanai and T. Amari, Rheol. Acta 34, 303 (1995).

[10] J. R. Melrose, J. H. vanVliet, and R. C. Ball, Phys. Rev. Lett. 77, 4660 (1996).
[11] A. Singh and P. R. Nott, J. Fluid Mech. 412, 279 (2000).

[12] C. O. Osuji and D. A. Weitz, Soft Matter (in press).

[13] M. C. Grant and W. B. Russel, Phys. Rev. E 47, 2606 (1993).

[14] R. G. Larson, The Structure and Rheology of Complex Fluids (Oxford University Press, Oxford, 1999).

[15] R. C. Sonntag and W. B. Russel, J. Colloid Interface Sci. 113, 399 (1987).

[16] R. Bandyopadhyay, D. Liang, H. Yardimci, D. A. Sessoms, M. A. Borthwick, S. G. J. Mochrie, J. L. Harden, and R. L. Leheny, Phys. Rev. Lett. 93, 228302 (2004).

[17] M. Bellour, A. Knaebel, J. L. Harden, F. Lequeux, and J.-P. Munch, Phys. Rev. E 67, 031405 (2003).

[18] L. Ramos and L. Cipelletti, Phys. Rev. Lett. 94, 158301 (2005).

[19] K. Dullaert and J. Mewis, J. Colloid Interface Sci. 287, 542 (2005).

[20] B. J. Maranzano and N. J. Wagner, J. Rheol. 45, 1205 (2001). 